\title{
Spatial and temporal processing in the auditory and visual modalities
}

\author{
JANET METCALFE, DORIS GLAVANOV, and MARTHA MURDOCK \\ University of Toronto, Toronto, Ontario MSS IAI, Canada
}

\begin{abstract}
Three experiments tested the idea that auditory presentation facilitates temporal recall whereas spatial recall is better if the input modality is visual. Lists of words were presented in which the temporal and spatial orders were independent, and instructions to the subjects determined whether recall would be given in a spatial or temporal order. In all three experiments, a significant interaction between the input modality and the type of recall was found, such that visual presentation resulted in superior recall over auditory presentation in the spatial conditions and auditory presentation yielded superior recall to visual in the temporal conditions. The present results contradict an earlier study by Murdock that showed that auditory presentation resulted in better performance than visual presentation in a nominally spatial task. An explanation for the discrepancies between the results of that study and the present one is presented.
\end{abstract}

In their review of verbal learning and memory, Tulving and Madigan (1970) discussed at length the retention differences that are often found between visually and auditorily presented items. They concluded the discussion by saying: "Why have we devoted so much space to the modality effects? The main reason is that they necessitate the rethinking of many problems and the rewriting of many theories. Auditory and visual information may be processed quite differently, and some phenomena may be entirely peculiar to one or the other modality. Understanding these differences seems to be a partial prerequisite to understanding human memory" (Tulving \& Madigan, 1970, p. 460). The difference between modalities that has often been demonstrated is that auditory presentation results in superior performance relative to visual presentation, particularly for the last few items presented (Craik, 1969; Crowder \& Morton, 1969; Murdock, 1968; Murdock \& Walker, 1969; and see also Penney, 1975). This finding is generally referred to as the modality effect. In this paper, we investigate further the processing differences that are found depending upon modality by varying the processing required by the task. The auditory and visual input modalities are compared in both a spatial and temporal recall task.

The authors would especially like to thank Bennet B. Murdock,

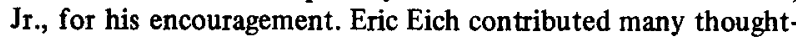
ful comments. The research was facilitated by a Natural Sciences and Engineering Council of Canada postgraduate scholarship awarded to the first author, NRC Grant APA 146 to Bennet B. Murdock, $\mathrm{J}_{\mathrm{r}}$, and NRC Grant A8632 to Endel Tulving. This article was based on a master's thesis submitted by the first author to the Department of Psychology at the University of Toronto. Some aspects of this research were presented at the annual convention of the American Psychological Association in 1978.
Several explanations of the modality effect would seem to indicate that there should be an auditory advantage regardless of the demands of the task. For instance, one explanation postulates that verbal material is coded phonemically and that translation into a phonemic code requires an extra operation if the input is visual, but not if it is auditory (Posner, 1967; Sperling, 1967). As long as the materials are verbal, this extra operation in the visual condition should result in recall inferior to that found in the auditory condition. Another theory of the modality effect proposes that there exist precategorical acoustic and visual stores. The information in these stores may subsidize the information in the common categorical store to the extent that the precategorical information persists. The auditory superiority that is usually found is explained in terms of a longer persistence of the information in the acoustic than in the visual store (Crowder \& Morton, 1969; Morton, 1970). These theories indicate that if any modality-specific difference occurs in recall, it should be in favor of the auditory modality.

There is some evidence that suggests that auditory input should not facilitate recall in a spatial task, however. Healy (1977) demonstrated that acoustic confusions were unimportant in a spatial recall task, compared with a similar temporal task (Bjork \& Healy, 1974), in which acoustic confusions were shown to play an important role. Acoustic confusions may be taken as an index of the importance of phonemic information. Watkins, Watkins, and Crowder (1974) have found that phonemic similarity attenuated the modality effect. If the modality effect is due to the utilization of the phonemic information in the auditory modality but that information is irrelevant in a spatial recall task, then it would be expected that there should be no modalityspecific difference in spatial recall.

There is also some reason to suppose that visual 
superiority should result when spatial recall is required. The possibility exists that processing in the auditory modality may be temporal in nature, whereas the visual modality is spatial (Murdock, 1969, 1974; Nilsson, Wright, \& Murdock, 1975; Paivio, 1971). As Murdock (1969) has argued, it may be the case that most, if not all, of the tasks that have been used to investigate the modality effect either have been explicitly temporal or could be performed by implementing a temporal organization. Murdock (1969) investigated the idea that visual input may result in better performance than auditory input if the task is spatial. Although his results showed superior auditory recall, we do not consider them to be definitive, for reasons that will be outlined shortly. O'Connor and Hermelin (1972) have demonstrated that there may be some preference for spatial organization with visual presentation and for temporal organization with auditory presentation.

\section{EXPERIMENT 1}

Experiment 1 tests the hypothesis that visual presentation facilitates spatial recall whereas auditory presentation facilitates temporal recall. Since the experiment tests a hypothesis similar to that proposed by Murdock (1969), a brief review of his experiment is in order.

In Murdock's (1969) study, subjects sat in front of a wall on which eight numbered loudspeakers were arranged in a circle. In the visual condition, slides of words were projected, one at a time, onto the grill cloths of the speakers, in random order. In the auditory condition, a tape that was channel controlled was played. The sound of any particular word was played through only one speaker. As in the visual condition, the spatial position of any word was randomly assigned without replacement. Because it was difficult to localize sounds presented in this manner, blank slides were projected onto the grill cloths of the appropriate loudspeakers in synchrony with the auditory presentation. A probe technique was used to elicit recall. Either the speaker number was used as a cue for the appropriate word or the word was given as a cue for the speaker number. Even though the list items were presented spatially, auditory presentation resulted in superior recall over visual presentation.

It would seem that this experiment supports the contention that auditory presentation results in recall superior to visual presentation regardless of the demands of the task. However, in an effort to investigate the processing that had occurred in the task, Murdock (1969) also analyzed errors. If subjects had processed the items in a spatial format, it would be expected that words that had been spatially near to each other would be most likely to be confused. This turned out not to be the case. If a word was not recalled in its correct spatial position, it was equally likely to be recalled in any of the other positions, irrespective of spatial distance. However, when the errors were analyzed in terms of their temporal input order, systematic results were found. When a word was not recalled in the correct position, it was most likely to be given in a temporally adjacent position. Thus, temporal, but not spatial, distance had an influence on recall. The conclusion that Murdock reached was that the lists in both modalities had been ordered temporally, not spatially. This finding has implications in terms of modality differences. It suggests that the visual input modality is not necessarily predisposed toward spatial processing. However, since processing apparently was not spatial in Murdock's (1969) study, the finding does not answer the question of whether auditory superiority results when subjects do process spatially.

In the present experiment, we attempted to avoid certain characteristics of Murdock's (1969) study that may have allowed subjects to use only the temporal input order. It seems likely that the number indicating the position of the loudspeaker was memorized along with the target word and that the verbal pairs, each consisting of a position number and an item, were maintained in their temporal order. To perform the task, no spatial information was required. To minimize dependence on this strategy, no verbal labels were assigned to the spatial positions in the present experiment. Also, subjects were instructed to recall the words in the positions in which they had occurred, rather than to recall the items attached to position numbers. Also, in Murdock's (1969) study, the presentation of visual cues in the auditory condition-the synchronous blank slides-may have influenced the results. It was possible for subjects to visually process the uniquely spatial information in both the visual and the auditory conditions. Even if visual-spatial information was retained more effectively than auditory-spatial information, this difference may not have been apparent in Murdock's results, since the spatial information might have been processed visually in both conditions. No visual-spatial markers were presented in the auditory conditions of the present study. To avoid perceptual problems, only three spatial positions were used.

A comparable temporal task was also included in the present design, allowing an evaluation of an attenuation of the modality effect in the spatial condition.

\section{Method}

Auditory and visual input modalities were crossed with spatial and temporal recall in a within-subjects design. Six lists of words were presented to each subject in each of the four treatment combinations. The lists in each combination were presented as a block. Each list consisted of 12 words randomly selected from the Toronto Word Pool, a collection of 1,040 common two-syllable words with contractions, archaic words, homophones, and proper nouns excluded. Each list was randomly divided into four "chunks" of 3 words each. The words were presented at a 1-sec rate, with a pause of $2 \mathrm{sec}$ separating one chunk from another. A bell in the auditory condi- 
tions and a row of question marks in the visual conditions signaled the end of the list. Within each chunk, there were three temporal positions (first, second, and third) and three spatial positions (left, right, and center). The spatial positions were randomly assigned without replacement within each chunk, resulting in the temporal and spatial positions being independent. The difference between the spatial and temporal levels depended, not on the lists, but on the instructions to the subjects.

Subjects were tested individually in $1-\mathrm{h}$ sessions. They were given booklets providing, for each list, four rows and three columns in which to write their recall. In the temporal conditions, they were instructed to write the first word in each chunk in the left column, the second in the center column, and the third in the right column. To do this task, where the item was presented could be ignored and only when it occurred needed to be considered. In the spatial conditions, subjects were instructed to put words that occurred on the left side of the screen or in the left earphone in the left column, words that occurred on the right side or in the right earphone in the right column, and center items in the center column. Recall was not necessarily completely serial; that is, as long as an item was in the correct position, it was scored as correct. Also, subjects were informed that they need not put the first chunk in the first row, second in the second, and so on. Within the confines of this design, correct chunk ordering could not be demanded without causing an imbalance in the amount of spatial and temporal information required. Before each condition, subjects paraphrased the instructions to the experimenter until the latter was convinced that the former understood the task. Recall was subject paced.

To control for practice effects, the order of presentation of treatment combinations was counterbalanced, with the restriction that a subject be required to change type of recall (i.e., spatial or temporal) only once during the session. This restriction was implemented to minimize confusion about the task. Identical word lists, with the identical spatial and temporal positioning, were also presented equally often in each treatment combination. This was done to eliminate, so far as possible, artifactual differences in the scores of any condition due to the varying difficulty of materials.

The total design of the experiment was a 2 by 2 by 6 by 4 by 3 factorial, in which the factors were, respectively, modality (auditory or visual), type of recall (spatial or temporal), lists, chunks (or quarter lists), and positions (within chunks). Periods (or order of presentation of treatment combinations) and word sets were also included as between-subjects control factors.

The auditory lists were recorded by a female on a fourchannel Sony tape recorder (Model TC850-4). The spatial positioning was achieved by redubbing the original monaural recording to another tape recorder and changing the balance control between each word to the appropriate position. When the word was from the left, all the sound was in the left ear, and when it was from the right, the sound was in the right ear; in the center combination of left and right, the sound seemed to subjects to be located in the center of the head. Subjects, seated in a sound booth, heard the auditory presentation via headphones. The visual lists were typed in uppercase and presented by means of an IBM Selectric typewriter, advanced by a solenoid controlled by a Hunter timer. Since there could be no precise demarcation of the border between positions in the auditory condition, none was presented in the visual condition. Each spatial location was eight letters wide, the width of the largest word used. Subjects, seated in a soundproof booth, viewed the presentation on closed circuit TV.

The subjects were 19 undergraduate and graduate volunteers from the University of Toronto. Some subjects received a bonus point in an introductory psychology course for participating and presenting a short paper on this experiment. Three subjects were eliminated, leaving 16 subjects. Two subjects had difficulty shifting conditions. One subject was dropped because of an inability to perceive the auditory-spatial positions. Among the remaining 16 subjects, there were no known hearing deficiencies, and all subjects reported that they could perceive the auditory positions.

\section{Results}

The data for the analyses that follow were arcsine transformed, although an analysis of variance was also conducted on the raw proportions and, in general, the transformation had no effect upon the significance levels. The data were collapsed across lists in order to obtain proportions. The untransformed proportions are presented in the tables and figures that follow. The alpha level chosen for significance was $p<.05$.

The interaction between the type of recall (either spatial or temporal) and input modality (either auditory or visual) was significant $[\mathrm{F}(1,15)=17.89, \mathrm{MSe}=.843]$ and is illustrated in Figure 1. A post hoc test, Tukey's "honestly significant differences" (HSD) test (Kirk, 1968 ), revealed that (1) in the spatial condition, visual input resulted in better recall than did auditory input, (2) in the temporal condition, auditory input resulted in better recall than visual, (3) there was no difference between the visual-temporal and visual-spatial means, and (4) there was a difference between the auditorytemporal and auditory-spatial means.

There was no main effect of modality $(F<1)$. There was an interaction between chunk and modality $[F(3,45)=5.86, \mathrm{MSe}=.423]$, as is illustrated in Figure 2. Tukey's HSD test revealed that the locus of this

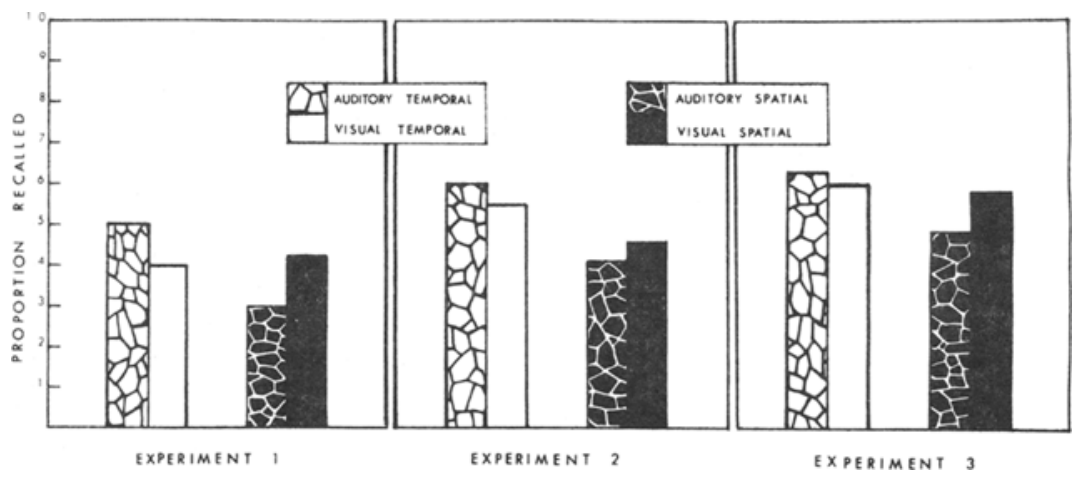

Figure 1. The interaction between type of recall and input modality. 


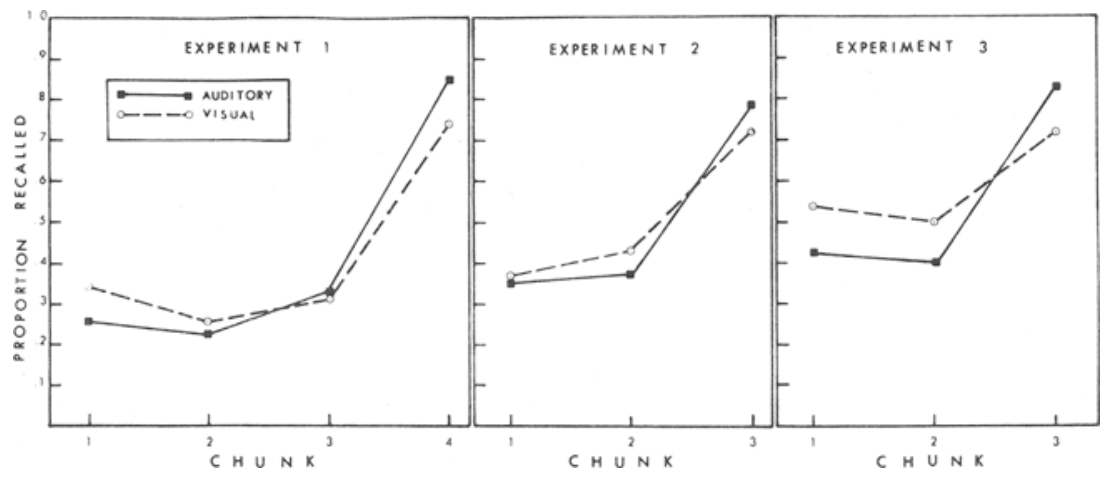

Figure 2. The interaction between input modality and chunk.

interaction was in the last, or recency, chunk, in which auditory presentation resulted in recall superior to visual presentation. A more lenient test (Fisher's least significant difference test; Kirk, 1968) showed that visual superiority was found in the first chunk, although with Tukey's more stringent criterion this difference missed significance.

The three-way interaction among modality, type of recall and chunk failed to reach significance $[F(3,45)=$ $1.44, \mathrm{MSe}=.438]$. The means may be inspected in Table 1. There was a main effect of chunk $[F(3,45)=$ 123.72, MSe $=.699]$ such that a bowed chunk-position curve was found, corresponding to the usual serial position curve.

There was a main effect of type of recall $[F(1,15)=$ 20.25 , MSe $=.588$ ], showing temporal superiority, as has been found by a variety of investigators (e.g., Healy, 1975b; Mandler \& Anderson, 1971). In this experiment, there was also an interaction between the type of recall and chunk $[\mathrm{F}(3,45)=4.75, \mathrm{MSe}=.313]$. The proportions recalled for each of the four chunks for temporal

Table 1

The Interaction Among Modality, Type of Recall, and Chunk

\begin{tabular}{|c|c|c|c|c|}
\hline \multirow[b]{2}{*}{ Condition } & \multicolumn{4}{|c|}{ Chunk } \\
\hline & 1 & 2 & 3 & 4 \\
\hline & \multicolumn{4}{|c|}{ Experiment 1} \\
\hline $\begin{array}{l}\text { Auditory-Temporal } \\
\text { Visual-Temporal } \\
\text { Auditory-Spatial } \\
\text { Visual-Spatial }\end{array}$ & $\begin{array}{l}.330 \\
.323 \\
.188 \\
.396\end{array}$ & $\begin{array}{l}.243 \\
.198 \\
.132 \\
.215\end{array}$ & $\begin{array}{l}.476 \\
.344 \\
.177 \\
.295\end{array}$ & $\begin{array}{l}.962 \\
.733 \\
.733 \\
.767\end{array}$ \\
\hline Visual-Spatial & \multicolumn{4}{|c|}{ Experiment 2} \\
\hline $\begin{array}{l}\text { Auditory-Temporal } \\
\text { Visual-Temporal } \\
\text { Auditory-Spatial } \\
\text { Visual-Spatial }\end{array}$ & $\begin{array}{l}.421 \\
.450 \\
.291 \\
.288\end{array}$ & $\begin{array}{l}.433 \\
.488 \\
.317 \\
.367\end{array}$ & $\begin{array}{l}.942 \\
.729 \\
.638 \\
.713\end{array}$ & \\
\hline Visual-Spatial & \multicolumn{4}{|c|}{ Experiment 3} \\
\hline $\begin{array}{l}\text { Auditory-Temporal } \\
\text { Visual-Temporal } \\
\text { Auditory-Spatial } \\
\text { Visual-Spatial }\end{array}$ & $\begin{array}{l}.505 \\
.550 \\
.358 \\
.541\end{array}$ & $\begin{array}{l}.498 \\
.516 \\
.413 \\
.484\end{array}$ & $\begin{array}{l}.884 \\
.757 \\
.786 \\
.691\end{array}$ & \\
\hline
\end{tabular}

recall were $.32, .22, .41$, and .85 ; for spatial recall, the means were $.29, .17, .23$, and .74 . The temporal superiority was mainly in the recency chunks of the list.

Although the interaction between within-chunk position and type of recall did not reach significance, there was a trend that suggested that in the temporal recall conditions, the within-chunk position curve may have been more bowed than in the spatial recall conditions $[\mathrm{F}(2,30)=3.31, \mathrm{MSe}=.115, \mathrm{p}<.1]$. This pattern is shown in Figure 3.

The main effect of position within chunk was significant $[F(2,30)=8.41, \mathrm{MSe}=.109]$ and can be inferred from Figure 3. Recall of the items in the first and third positions was better than recall of the item in the middle position.

Error analyses. The fact that an interaction between input modality and type of recall was found, itself, indicates that there was some difference in the type of processing that was done in the spatial and temporal conditions. The within-chunk position curves that were somewhat more bowed in the temporal conditions than in the spatial conditions also indicate that there was some difference in processing in the two conditions. A similar result found by Healy (1975b) has been interpreted as evidence that a spatiotemporal pattern code is used in the spatial conditions. In order to further assess whether or not the spatial lists were in fact coded spatially and the temporal lists temporally, an analysis of the transposition errors was conducted. This analysis was important in order to reconcile the differences between the present results and Murdock's (1969) findings.

A transposition may be said to have occurred if an item is recalled in a position other than its correct position. If items are ordered along a particular dimension, then one would expect to find more transpositions between items that were next to each other on that dimension (i.e., adjacent transpositions) than between items that are further removed from each other (i.e., remote transpositions). Following the technique introduced by Murdock and Vom Saal (1967), each entire 


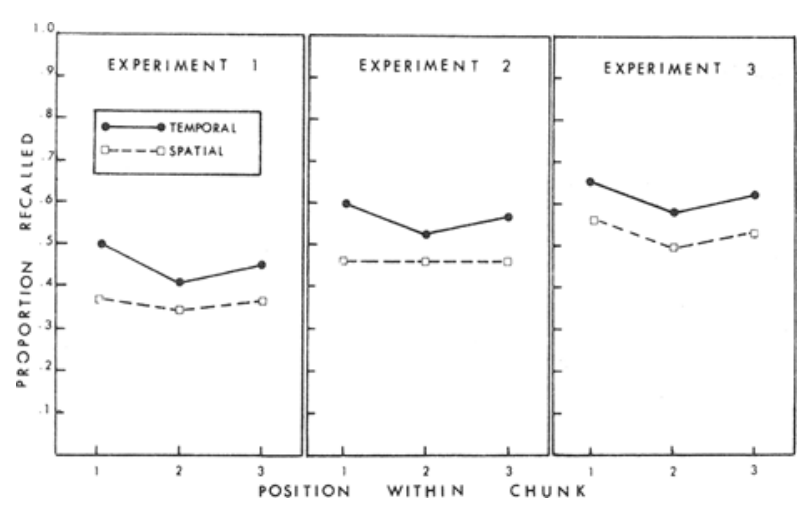

Figure 3. The interaction between type of recall and position within chunk.

triad or chunk was classified as correct order, adjacent transposition, or remote transposition. If the correct order for a particular triad was $A B C$, an adjacent transposition would be $A C B$ or $B A C$ and remote transpositions would be $\mathrm{BCA}, \mathrm{CAB}$, and $\mathrm{CBA}$. If an item error (i.e., an omission or intrusion) occurred in a triad, the triad was considered to be a no-transposition, adjacent, or remote triad, according to the classification detailed in Murdock and Vom Saal (1967). The no-transposition case was considered to be the correct spatial order within a particular chunk in the spatial conditions and the correct temporal order in the temporal conditions. Murdock and Vom Saal have argued that if there are more adjacent than remote transpositions, "the frequency difference can serve as a guarantee of a genuine serial order effect" (1967, p. 138). The frequency of remote transpositions expected by chance, using this classification, is greater than the expected frequency of adjacent transpositions (whether triads containing item errors are included or not). Since the spatial and temporal orders that were presented in the lists were uncorrelated, if the spatial lists were processed only temporally, then more remote than adjacent transpositions should be found, as would be expected by chance. If the spatial information was used, more adjacent than remote transpositions should occur.

Table 2 shows the conditional transposition probabilities, based on those triads in which at least one word was recalled. In all four treatment combinations, there were more adjacent than remote transpositions. The expected probabilities in the table are the chance values that would be expected if all possible permutations and combinations of item and order errors were equally likely. The finding that there were more adjacent than remote transpositions in the spatial conditions indicates that the spatial information was utilized in the spatial conditions. In the temporal conditions, there was a slight bias toward adjacent transpositions, attributable to the fact that the first and last items in each triad were more likely to be recalled (see Figure 3). This bias is less important in the spatial conditions, since the level of recall in those conditions was more nearly equal across the triad. It may be concluded that the reason for the difference between the present results and those of Murdock (1969) is that in the present case, subjects were using spatial information to perform the task, whereas in the earlier study, they were not.

\section{Discussion}

In this experiment, it was found that when spatial recall was required, the visual input modality resulted in performance superior to that of the auditory modality and when temporal recall was required, auditory input resulted in superior recall. This is in contrast to Murdock's (1969) study, in which auditory presentation resulted in spatial recall superior to that of visual presentation. However, in Murdock's experiment, no evidence could be found for spatial processing, even though nominally spatial recall was required. In the present experiment, the transposition frequencies indicate that subjects were using the spatial information in their processing of the lists. It would appear that the differences between the present experiment and Murdock's experiment are attributable to differences in the extent to which spatial information was utilized.

\section{EXPERIMENT 2}

The second experiment was run as a replication of the first, with some minor changes. The most important difference was the inclusion of a test to ensure that subjects were able to localize the auditory-spatial locations. If subjects in the first experiment had been unable to discriminate between the auditory-spatial positions, it might be expected that performance would suffer. Therefore, in this experiment, a test was given to be sure

Table 2

Conditional Transposition Probabilities

\begin{tabular}{lccc}
\hline \multicolumn{1}{c}{ Condition } & \multicolumn{3}{c}{ Type of Transposition } \\
\cline { 2 - 4 } Auditory-Temporal & None & Adjacent & Remote \\
\hline Visual-Temporal & .842 & .112 & .047 \\
Auditory-Spatial & .719 & .195 & .085 \\
Visual-Spatial & .552 & .284 & .163 \\
& .807 & .144 & .048 \\
Auditory-Temporal & .883 & .073 & .043 \\
Visual-Temporal & .811 & .151 & .038 \\
Auditory-Spatial & .581 & .232 & .187 \\
Visual-Spatial & .732 & .153 & .116 \\
& & Experiment 1 \\
& .854 & .115 & .031 \\
Auditory-Temporal & .862 & .109 & .028 \\
Visual-Temporal & .733 & .185 & .082 \\
Auditory-Spatial & .822 & .136 & .042 \\
Visual-Spatial & .212 & .364 & .424 \\
Expected & & & \\
\hline
\end{tabular}


the auditory locations could be perceived as well as the visual could. The list length was also decreased from four to three chunks.

\section{Method}

Although the basic method used was the same as that in Experiment 1, a number of small changes were made. Because of time constraints, the list length was reduced to nine items, or three chunks. The recall time was experimenter paced at $40 \mathrm{sec} /$ list, rather than subject paced. Subjects received one practice list before each condition shift. This allowed them to change strategies on lists that were not scored. It also eradicated the difficulties in shifting conditions that were found in Experiment 1 . Because of session length constraints, this allowed time for only five lists per condition. As before, there were four sets of lists. In this experiment, the sets were randomly assigned to treatment combinations, and subjects were randomly assigned to a particular order of treatment combinations. The person who recorded the auditory conditions was male rather than female.

The most important addition in Experiment 2 was a percep. tion test at the beginning and end of the session. Subjects received three auditory and three visual lists at the beginning and end of the session, in which spatial perception was tested. The order of presentation of the modalities and the set of words used was randomly determined. Subjects were instructed for each chunk to write a " 1 " in the position of the first word, a " 2 " in the position of the second word, and a " 3 " in the position of the third. The lists were structurally the same as those used in the memory part of the experiment; only the subject's task differed. There was a practice perception list in each modality to which the subject simply attended.

Subjects were selected on the basis of the perception test. The justification for selecting subjects was that even a small imbalance in hearing might cause enough difficulty in the perception of the auditory locations to make such a subject's data questionable. When it is demonstrated that no perceptual differences exist, the argument that any memory differences between the auditory and visual conditions are really perception differences becomes less convincing. Twenty-three subjects were tested. Two subjects were eliminated for procedural reasons having nothing to do with perception. Five subjects were eliminated because they failed to meet the criterion that was decided upon for acceptance, which was that they could make no more than one more error on the auditory perception test than they made on the visual perception test. The remaining 16 subjects made a total of 12 errors on the perception test (of a possible 412); 6 were on the visual lists and 6 were on the auditory lists.

\section{Results}

The interaction of interest, between input modality and type of recall, was again significant $[F(1,15)=8.14$, $\mathrm{MSe}=.338]$ and is presented in the center panel of Figure 1. Tukey's HSD test revealed that recall was better in the auditory-temporal condition than in the visual-temporal condition; the mean for the auditorytemporal condition was greater than that of the auditory. spatial condition. In this experiment, the difference between the visual- and auditory-spatial conditions did not reach significance. However, the overall pattern of results was the same as in the previous experiment.

As before, temporal recall was better than spatial recall $[\mathrm{F}(1,15)=32.91, \mathrm{MSe}=.646]$. There was an effect of chunk $[F(2,30)=42.95$, MSe $=1.408]$ such that the last chunk was recalled better than the first two chunks.
The interaction between input modality and chunk was not significant $[F(2,30)=2.45, \mathrm{MSe}=.545]$, although the means, which are presented in Figure 2, are in the same direction as in the first experiment. The triple interaction among input modality, type of recall, and chunk was significant $[\mathrm{F}(2,30)=6.13, \mathrm{MSe}=.597]$ and is presented in Table 1. Tukey's test revealed that this interaction is entirely attributable to the auditory superiority in the final chunk of the temporal recall condition. So, with temporal recall the modality effect was found, whereas with spatial recall it was not.

There was an interaction between the type of recall and position within chunk $[F(2,20)=8.30, \mathrm{MSe}=.216]$ such that in the temporal conditions the within-chunk position curves were U-shaped, whereas in the spatial conditions they were flat. This interaction, illustrated in Figure 3, replicates Healy's (1975b) findings. Finally, there was an interaction between chunk and position within chunk $[F(4,60)=3.74, \mathrm{MSe}=.177]$.

No other effects or interactions were significant. In particular, there was no main effect of input modality $(F<1)$.

Error analyses. Table 2 shows that, as was the case in the first experiment, there were more adjacent than remote transpositions in all four treatment combinations. As in the first experiment, some spatial information appears to have been used in the present experiment.

In an effort to gain some insight into the reason for the interaction that was found between input modality and type of recall, a further error analysis was conducted on the relation between item and order information. There is reason to suppose (see Estes, 1972) that there should be a positive but not perfect correlation between the loss of item information and the loss of temporal order information. What little evidence there is on the relation between item and spatial order information indicates that the two should be uncorrelated. Healy (1975a) showed that when only the spatial order information had to be retained, there was no loss over time. Since there is a loss of temporal order information and item information over time, it would be expected that temporal order and item information should be correlated, whereas spatial order and item information may be uncorrelated.

On the other hand, input modality has not been varied in comparisons of spatial and temporal recall. It is conceivable that there may be a compatibility between the type of processing required and the input modality. One way in which this compatibility might be manifested is in terms of the correlation between item and order information. It might be the case that the correlation between item and order information is modality specific.

To investigate the relation between the loss of item information and the loss of order information, each triad from the transposition analysis was entered into a 2 by 2 classification. A triad either did or did not have a 
transposition, and a triad either did or did not have an item error (either an intrusion or an omission). In order to analyze these data statistically, the observations were treated as if they were independent. Each of the four treatment combinations were separately analyzed by means of a chi-square test. The phi values in each of the treatment combinations that describe the strength of the correlation between item and order loss were then used to compute the differences between correlations for each pair of treatment combinations.

Table 3 presents the conditional probabilities of item errors, given either a transposition or no transposition, in each of the four treatment combinations, as well as the phi and chi-square statistics. As can be seen from the table, there was a positive correlation between item and order information in the temporal conditions, but not in the spatial conditions, of Experiment 2. A comparison of the correlations revealed no difference between the auditory-temporal and visual-temporal phi values. There was no difference between the auditory-spatial and visual-spatial correlations. The strength of the correlation between item and order information was greater in both of the temporal conditions than in either of the spatial conditions $\left[\mathrm{z}_{(\mathrm{AT}-\mathrm{AS})}=3.03 ; \mathrm{z}_{(\mathrm{AT}-\mathrm{VS})}=4.72\right.$; $\left.\mathrm{z}_{\text {(VT-AS) }}=2.28 ; \mathrm{z}_{\text {(VT-VS) }}=2.16\right]$. It appears that the item-order correlation breaks down in terms of type of recall, but that input modality, in this experiment at least, had no influence on the relation.

In Experiment 1, all four conditions showed a positive correlation between the loss of order information and the loss of item information, although the strength of the relation was greater in the temporal than in the spatial conditions, as in Experiment 2. There was no difference between the correlations for the auditorytemporal and visual-temporal conditions, nor was there any difference between the correlations for the auditory-

Table 3

The Relation Between Item and Order Information

\begin{tabular}{lcccc}
\hline & \multicolumn{4}{c}{ Probability* } \\
\cline { 2 - 4 } & $\mathrm{NT}$ & $\mathrm{T}$ & $\phi$ & $\chi^{2}$ \\
\hline & \multicolumn{4}{c}{ Experiment 1} \\
Auditory-Temporal & .393 & .864 & .344 & $32.93 \dagger$ \\
Visual-Temporal & .477 & .829 & .328 & $29.15 \dagger$ \\
Auditory-Spatial & .514 & .643 & .187 & $8.96 \dagger$ \\
Visual-Spatial & .573 & .769 & .158 & $6.77 \dagger$ \\
& \multicolumn{5}{c}{ Experiment 2} \\
Auditory-Temporal & .419 & 1.00 & .373 & $28.54 \dagger$ \\
Visual-Temporal & .529 & .90 & .296 & $18.59 \dagger$ \\
Auditory-Spatial & .487 & .494 & .069 & .94 \\
Visual-Spatial & .518 & .608 & .079 & 1.21 \\
& \multicolumn{4}{c}{ Experiment 3} \\
Auditory-Temporal & .323 & .887 & .406 & $80.13 \dagger$ \\
Visual-Temporal & .374 & .882 & .353 & $61.33 \dagger$ \\
Auditory-Spatial & .406 & .760 & .313 & $44.38 \dagger$ \\
Visual-Spatial & .401 & .600 & .154 & $11.30 \dagger$ \\
\hline
\end{tabular}

Note $-N T=$ no transposition; $T=$ transposition

*Conditional probability of an item error. spatial and visual-spatial conditions. Once again, input modality did not affect the correlation between the item and order information loss. There was a difference in the correspondence of item and order information, depending upon type of recall. While the difference between the visual-temporal and the auditory-spatial correlations was only marginally significant $(z=1.75$, $p<.1$ ), the differences between the auditory-temporal and auditory-spatial conditions $(z=1.96)$, the auditorytemporal and visual-spatial conditions $(\mathrm{z}=2.36)$, and the visual-temporal and visual-spatial conditions $(\mathrm{z}=2.11)$ were significant.

\section{Discussion}

Although the interaction between modality and type of recall was again significant, we were still not satisfied that the perception of the auditory-spatial positions was without influence. Therefore, in the third replication, an attempt was made to improve the method of presentation of the auditory stimuli. Instead of presenting the words via headphones and mixing the left and right channels to form a central position, the words were presented by means of three speakers. This method of presentation allows the subjects to make better use of the onset time of stimuli between ears. This cue (as well as the relative volume of the stimuli between ears) is known to be important in the localization of sounds (B'́késy, 1967). This method of presentation has the disadvantage of potentially providing subjects with visual cues in the auditory conditions. If these visual cues were used, however, they would tend to decrease rather than increase the chance of finding a visual advantage in spatial recall.

It was also thought that the output modality might have influenced the results. In Experiments 1 and 2, recall was written. Therefore, in this replication, output modality, either written or spoken, was manipulated as a within-subjects but between-sessions variable.

In the first two experiments, subjects were free to write their responses in any order they chose. As long as the correct item was placed in the correct position, no constraint was placed on the order in which the responses themselves were given. According to Healy (1977), one strategy that may be used in the spatial task is to employ a spatiotemporal pattern. In the present experiment, subjects were instructed to recall the items in each chunk in the spatial conditions in a left-to-right order. This constraint may make it more difficult to employ the temporal aspect of the spatiotemporal pattern and put more emphasis on the purely spatial aspect of spatial recall.

\section{EXPERIMENT 3}

\section{Method}

The method was essentially the same as that used in Experiment 2 , except that the auditory stimuli were presented by means of wall-mounted loudspeakers. The subject was seated in the center of a small soundproof chamber with the left speaker 
directly on the left, the right speaker on the right, and the center speaker directly in front. The words were recorded by a female onto the rear channel of the Sony four-channel TC850-4. The individual words were then transferred to Channel 1,2 , or 3 (which corresponded to the left, center, and right speakers) by using the synchrotracking capabilities of the tape recorder.

In order to test subjects' perception of the auditory-spatial locations, three lists were presented to each subject at the beginning of the first session. The subjects were asked to point to the loudspeaker from which the words were presented, as they were presented. The experimenter noted any errors. This method of perception testing was implemented to minimize any possible memory load in the perception test.

Twelve subjects were each tested for two sessions. Half of the subjects had written recall on the first session and half had spoken recall. In order to recall aloud, subjects were instructed to recall the left (or first in the temporal conditions) word first, the center (or second) word second, and the right (or third) word third, one chunk at a time. In the event that a subject had forgotten the word in a particular position, he or she said "blank." The responses were taped. Written recall in this experiment was also strictly serial, within each chunk.

The order of treatment combinations was randomly assigned, with the restriction that the same subject never received the same order in the two sessions. There was a practice list before each change in condition.

\section{Results}

None of the 12 subjects who were tested made any errors on the localization test. The method of presentation used in the present experiment appears to have eliminated any perceptual difficulties that may have been present in the previous experiments.

In this experiment, the interaction between input modality and type of recall was again found to be significant $[F(1,11)=10.80$, MSe $=.324]$ and is illustrated in Figure 1. Tukey's HSD test revealed that the difference between the visual-spatial and the auditory. spatial conditions was significant. The difference between the auditory-spatial and auditory-temporal conditions was also significant. The difference between the auditorytemporal and visual-temporal conditions did not reach significance, however, and there was no difference between the visual-spatial and the visual-temporal means.

As in the first two experiments, there was no main effect of modality. Temporal recall was better than spatial recall $[F(1,11)=34.42, \mathrm{MSe}=.291]$. None of the interactions with response modality was significant or even showed a trend.

There was a main effect of position within chunk $[F(2,22)=17.57, \mathrm{MSe}=.132]$. However, in contrast to the second experiment, there was no hint of an interaction between type of recall and the position within chunk $(F<1)$. Both the temporal and spatial conditions showed bow-shaped within-chunk position curves, as the temporal conditions had but the spatial conditions had not in the first two experiments. The means are presented in Figure 3.

There was a main effect of chunk $[F(2,22)=8.18$, $M S e=5.927$ ] that showed that the third, or recency, chunk was recalled better than the two prerecency chunks. There was also an interaction between input modality and chunk $[\mathrm{F}(2,22)=8.23, \mathrm{MSe}=.599]$, which is illustrated in Figure 2. Tukey's HSD test revealed auditory superiority in the last chunk and visual superiority in the first chunk. The visual advantage in the second chunk just missed significance.

There were no other significant effects or interactions in this experiment. The triple interaction among input modality, type of recall, and chunk was not significant $[\mathrm{F}(2,22)=1.07, \mathrm{MSe}=.275]$. The means in Table 1 show that with temporal recall, the usual auditory advantage was found in the recency chunk. In the spatial condition, the visual advantage showed up in the primacy part of the serial position curve. The net result, however, was no interaction.

Error analyses. As in the previous experiments, there were more adjacent than remote transpositions in all four treatment combinations, as is shown in Table 2. In this experiment, as in the previous experiments, subjects appear to have used the spatial information. The correlations between item and order information, presented in Table 3, suggest that the way in which the information was used may have been different in the present experiment, however. The correlations between loss of item information and loss of order information were significantly different from zero in all four treatment combinations. Although different from zero, the correlations between item and order information in the auditory-spatial, auditory-temporal, and visual-temporal conditions did not significantly differ from each other. There was a difference between each of these three conditions (auditory-temporal, visual-temporal, and auditory-spatial) and the item-order correlation in the visual-spatial condition $\left[\mathrm{z}_{(\mathrm{VS}-\mathrm{AT})}=3.89 ; \mathrm{z}_{(\mathrm{VS}-\mathrm{VT})}=\right.$ $\left.3.09 ; \mathrm{z}_{\text {(VS-AS) }}=2.42\right]$, such that the relation between item and order information was weaker in the visualspatial condition than in any of the other three conditions.

\section{Discussion}

In this experiment, once again, the interaction between input modality and type of recall was found. Since no subject made even a single error in localizing the auditory-spatial locations, it seems safe to assume that the difference in recall is not attributable to a perceptual difficulty in the auditory-spatial condition. The interaction between input modality and type of recall also does not appear to be influenced by output modality, which had no detectable effect in this experiment.

Although the overall pattern of results was the same in the present experiment as in the previous two, two small differences are notable. First, within-chunk position did not interact with type of recall. Second, the item-order correlation did not break down so neatly in terms of type of recall. It is possible that the constrained output order required in this experiment but not in the previous experiments is responsible for these differences. However, this did not appear to influence the robustness of the interaction of primary interest, that between input modality and type of recall. 


\section{CONCLUSION}

Since in three separate experiments an interaction between input modality and type of recall was found, it seems reasonable to acknowledge that the effect is real. The immediate question that comes to mind is why visual superiority was found in the spatial task in the present experiments, but not in Murdock's (1969) study. The most likely explanation is that in the earlier study subjects were not processing spatially, whereas in the present study they were. The transposition error analyses that were presented substantiate this explanation.

The finding of visual superiority in the spatial task and auditory superiority in the temporal task provides support for the idea of modality-specific memories (Murdock, 1974; Paivio, 1971). A difference in recall depending upon input modality was found not only in recency, but also in primacy. This suggests that the influence of the sensory characteristics of information may not be restricted to the very early stages of processing.

The precategorical acoustic store explanation of modality differences (Crowder, 1976, 1978; Crowder \& Morton, 1969), taken by itself, does not appear to predict the results found in the spatial conditions. The present results do not rule out the possibility of a precategorical acoustic store. They do indicate that there may be some other difference in the processing of auditory and visual information.

Drewnowski's (1980) proposal that in the auditory conditions there exists a "directional auditory trace" that enhances recall of the temporal order may also be consistent with the present results. According to Drewnowski, "whenever the auditory trace is available, its use in recall is obligatory" (1980, p. 192). This directional auditory trace would be expected to improve temporal recall. It might, however, impede spatial recall, since it would produce a tendency to recall the auditorily presented items in their temporal rather than spatial order.

\section{REFERENCES}

Békésy, G. Von. Sensory inhibition. Princeton, N.J: Princeton University Press, 1967.

Buork, E. L., \& Healy, A. F. Short-term order and item retention. Journal of Verbal Learning and Verbal Behavior, 1974, 13, 80-97.

CRAIK, F. I. M. Modality effects in short-term storage. Journal of Verbal Learning and Verbal Behavior, 1969, 8, 658-664. Crowder, R. G. Principles of learning and memory. Hillsdale, N.J: Erlbaum, 1976.
Crowder, R. G. Mechanisms of auditory backward masking in the stimulus suffix effect. Psychological Review, 1978, 85, $502-524$.

Crowder, R. G., \& Morton, J. Precategorical acoustic storage (PAS). Perception \& Psychophysics, 1969, 5, 365-373.

Drewnowski, A. Memory functions for vowels and consonants: A reinterpretation of acoustic similarity effects. Journal of Verbal Learning and Verbal Behavior, 1980, 19, 176-193.

Estes, W. K. An associative basis for coding and organization in memory. In A. W. Melton \& E. Martin (Eds.), Coding processes in human memory. Washington, D.C: Winston, 1972.

HeALY, A. F. Coding of temporal-spatial patterns in short-term memory. Journal of Verbal Learning and Verbal Behavior, $1975,14,481-495$. (a)

HeALy, A. F. Short-term retention of temporal and spatial order. Bulletin of the Psychonomic Society, 1975, 5, 57-58. (b)

Healy, A. F. Pattern coding of spatial order information in short-term memory. Journal of Verbal Learning and Verbal Behavior, 1977, 16, 419-437.

KIRK, R. E. Experimental design: Procedures for the behavioral sciences. Belmont, Calif: Brooks/Cole, 1968.

Mandler, G., \& Anderson, R. E. Temporal and spatial cues in seriation. Journal of Experimental Psychology, 1971, 90, 128-135.

Morton, J. A functional model for memory. In D. A. Norman (Ed.), Models of human memory. New York: Academic Press, 1970.

Murdock, B. B., JR. Modality effects in short-term memory: Storage or retrieval? Journal of Experimental Psychology, 1968, 77, 79-86.

Murdock, B. B., Jn., Where or when: Modality effects as a function of temporal and spatial distribution of information. Journal of Verbal Learning and Verbal Behavior, 1969, 8, 378-383.

Murdock, B. B., JR. Human memory: Theory and data. Potomac, Md: Erlbaum, 1974.

Murdock, B. B., Jr., \& Vom SaAL, W. Transpositions in shortterm memory. Journal of Experimental Psychology, 1967, 74, 137-143.

Murdock, B. B., JR., \& Walker, K. D. Modality effects in free recall. Journal of Verbal Learning and Verbal Behavior, $1969,8,665-676$.

Nilsson, L-G., Wright, E., \& Murdock, B. B., JR. The effects of visual presentation method on single-trial free recall. Memory \& Cognition, 1975, 3, 427-433.

O'Connor, N., \& Hermelin, B. Seeing and hearing in space and time. Perception \& Psychophysics, 1972, 11, 46-48.

Paivio, A. Imagery and verbal processes. New York: Holt, Rinehart, \& Winston, 1971.

Penney, C. G. Modality effects in short-term verbal memory. Psychological Bulletin, 1975, 82, 68-84.

Posner, M. I. Short-term memory systems in human information processing. Acta Psychologica, 1967, 27, 267-284.

SPE rLiNG, G. Successive approximations to a model for shortterm memory. Acta Psychologica, 1967, 2, 285-292.

Tulving, E., \& Madigan, S. A. Memory and verbal learning. Annual Review of Psychology, 1970, 21, 437-484.

Watkins, M. J., Watkins, O. C., \& Crowden, R. G. The modality effect in free and serial recall as a function of phonological similarity. Journal of Verbal Learning and Verbal Behavior, 1974, 13, 430-447.

(Received for publication February 13, 1980; revision accepted February 17, 1981.) 